\title{
Long-Term Use of Intravitreal Bevacizumab (Avastin) for the Treatment of Von Hippel-Lindau Associated Retinal Hemangioblastomas
}

\author{
Frank N. Hrisomalos ${ }^{1}$, Raj K. Maturi* ${ }^{*}, 2$ and Veena Pata $^{2}$ \\ ${ }^{1}$ Indiana University Department of Ophthalmology, Indianapolis, Indiana, USA \\ ${ }^{2}$ Vitreo-Retinal Service, Midwest Eye Institute, Indianapolis, Indiana, USA
}

\begin{abstract}
Retinal hemangioblastomas are the most common manifestation of Von Hippel-Lindau (VHL) disease [1-3]. While peripheral retinal hemangioblastomas may be treated by thermal laser treatment or cryotherapy, optic nerve and macular lesions are more difficult to treat $[4,5]$. Based on the theoretical benefit of administering anti-VEGF treatment, intra-vitreally administered bevacizumab (Avastin, a general pan-VEGF inhibitor) is attractive $[6,7]$.

Several short-term case series using ranibizumab (Lucentis, mAb fragment of bevacizumab with stronger affinity for VEGF-A) have shown it has promising but minimal success on most VHL-related hemangioblastomas [8, 9]. A comprehensive study by Wong et al. examined 5 patients over a period up to 61 weeks ( $47 \pm 14$ weeks) while Michels et al. examined one patient over a period of 4 months. Due to the short-term nature of these studies, we attempted long-term bevacizumab treatment over 60 months in a monocular subject with progressive visual loss due to a VHL associated macular and optic nerve hemangioblastoma. Over the treatment regimen of 15 injections, visual acuity improved 25 letters, OCT thickness improved from $646 \mathrm{um}$ to $424 \mathrm{um}$, and structural lesions stabilized while exudates and edema resolved.
\end{abstract}

Keywords: VHL, angioma, exudate, optic nerve glioma, bevacizumab, avastin, treatment, long-term.

\section{INTERVENTION}

A 33-year-old male with a long history of VHL associated tumors including benign cysts on liver, kidneys and cerebellum presented with multiple large retinal hemangioblastomas in his remaining right eye. We successfully treated a large peripheral hemangioblastoma with cryotherapy and a smaller equatorial lesion with thermal laser. However, significant macular thickening with moderate hard exudates were present due to an optic nerve lesion with extension towards the fovea (Fig. 1A). We treated the lesion with a total of 15 bevacizumab injections over a period of 60 months. Visual acuity (VA), appearance on fluorescein angiogram (FA), and optical coherence tomography (OCT) measurements were used as indicators of treatment success. VA was measured using a Reichert eye chart and was converted to visual acuity notation letters in Fig. (2).

\section{RESULTS}

Baseline VA was 20/100. Due to worsening vision, continued leakage on angiography and macular thickening, the patient underwent treatment as outlined in Fig. (2). At 12 months and after treatment with thermal laser therapy, cryotherapy and intravitreal kenalog, given the lack of biomicroscopic or angiographic response, the patient began receiving intravitreal bevacizumab (Avastin $1.25 \mathrm{mg}$ in 0.05 cc) treatments at regular intervals. At 24 month follow up,

*Address correspondence to this author at the Midwest Eye Institute, $200 \mathrm{~W}$. $103^{\text {rd }}$ Street, suite 1060 Indianapolis, IN 46290, USA; Tel: 317-817-1414; Fax: 317-805-4587; E-mail: rmaturi@indyretina.com
VA improved to $20 / 30$, the structural lesion stabilized and the exudates and edema resolved (Fig. 1B). The OCT showed only a partial improvement in thickness (646 um to $414 \mathrm{um}$ ) likely due to the presence of structural changes in the retina (Fig. 3A, B). On final examination, after a follow up of 60 months and 15 total bevacizumab injections, VA showed minimal regression to 20/40 and remained markedly better than VA on presentation. The structural lesion remained stable and no new exudates or edema were appreciated (Fig. 1C). OCT showed that thickness remained substantially decreased from initial presentation (Fig. 3C). No systemic or ocular adverse events related to any of the treatments were noted.

\section{DISCUSSION}

Macular and optic nerve lesions due to VHL associated hemangioblastomas have generally been difficult to treat. As one of the major mechanisms of disease is due to the poor regulation of VEGF (among others) production, using a panVEGF blocker over the long term is both logical and potentially helpful. To our knowledge, this is the longestterm study involving pan anti-VEGF therapy to treat VHL. While it is difficult to compare the results between the other two studies using VEGF suppression by pegaptanib or semaxanib with the current single case study, it is instructive to note that even the best subject in Wong's series (among the five total subjects) treated with pegaptanib did not achieve as significant a reduction in central macular thickness as our patient with bevacizumab did.

Wong et al. examined the effects of ranibizumab on five patients with VHL associated hemangioblastomas in a short- 


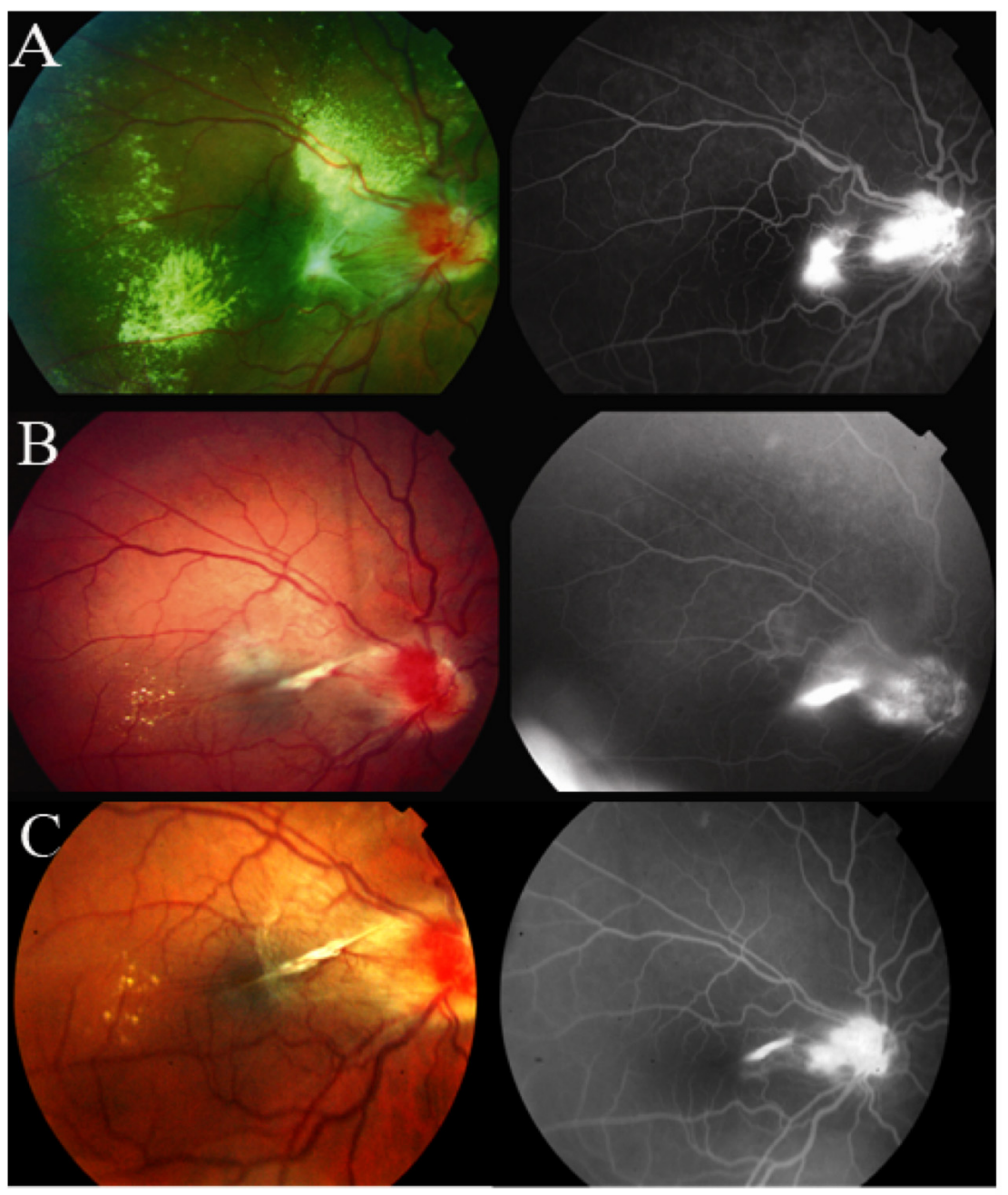

Fig. (1). Fundus Photographs and Fluorescein Angiograms at 0, 24, and 60 months.
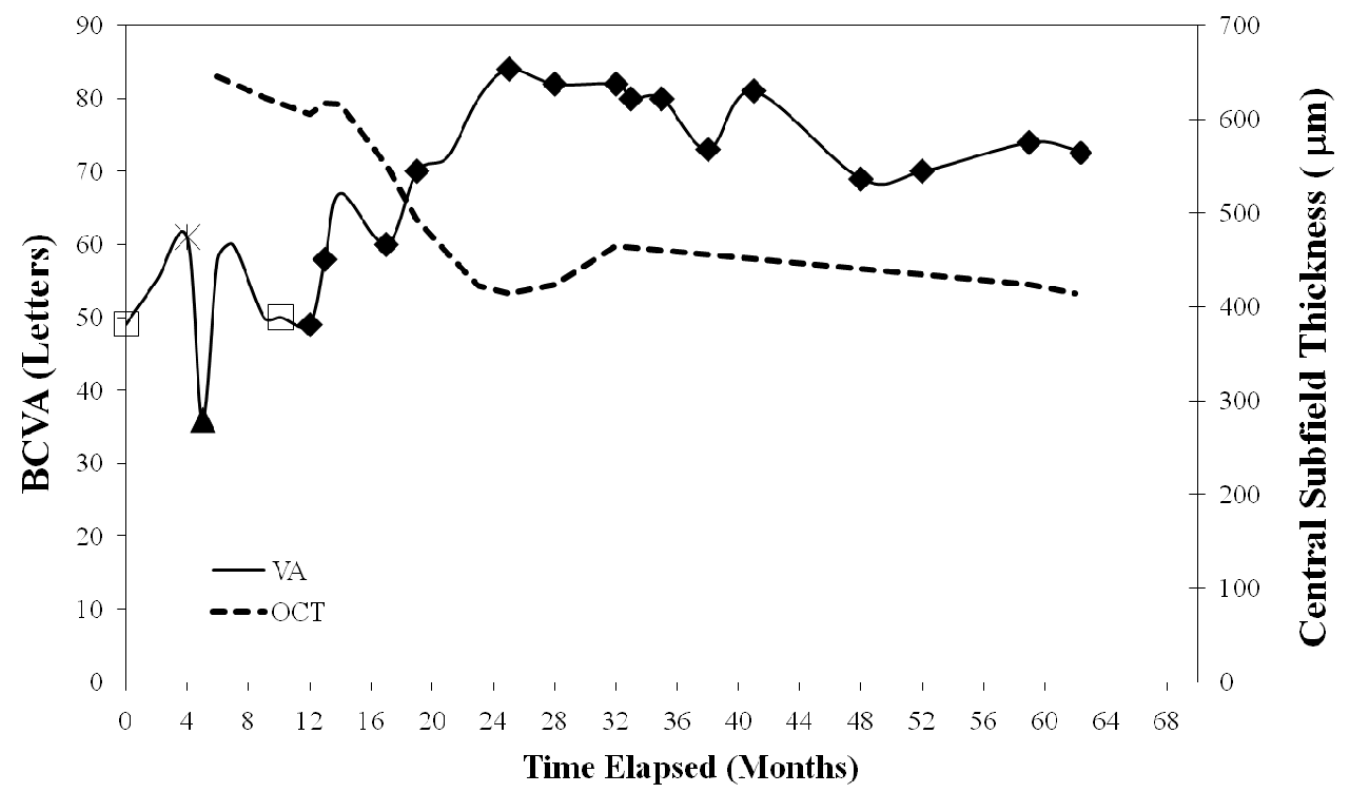

Fig. (2). A schematic representation at 0,24 , and 60 months of best-corrected visual acuity (BCVA) and central subfield thickness ( $\mu \mathrm{m})$, as measured by optical coherence tomography (OCT), with designated mode of treatment, OD. Open symbols indicate extra ocular treatments and closed symbols indicate intraocular treatments, and are as follows: $(\square)$ - Thermal laser therapy, $(\boldsymbol{\Delta})$ - Intravitreal Triamcinolone, $(*)$ Cryotherapy and ( $\bullet$ - Avastin. 

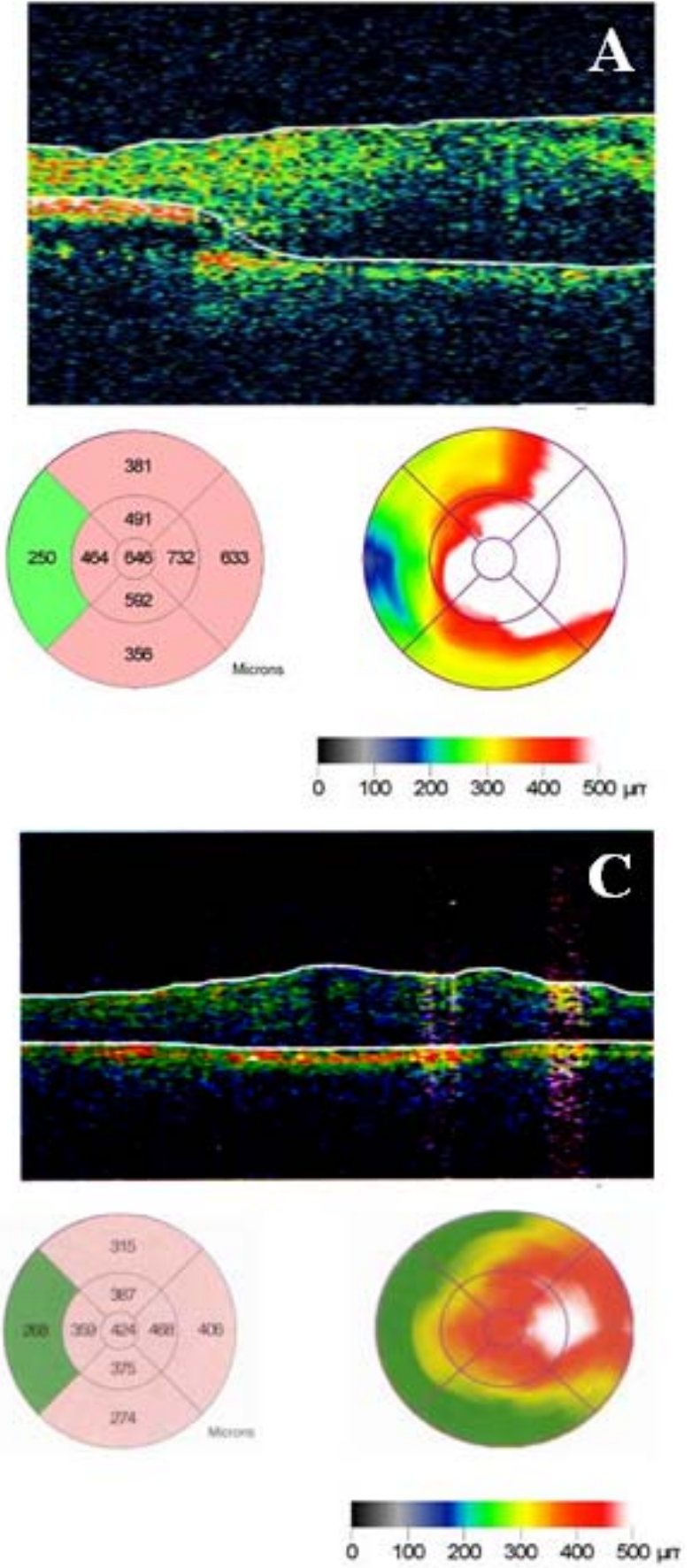
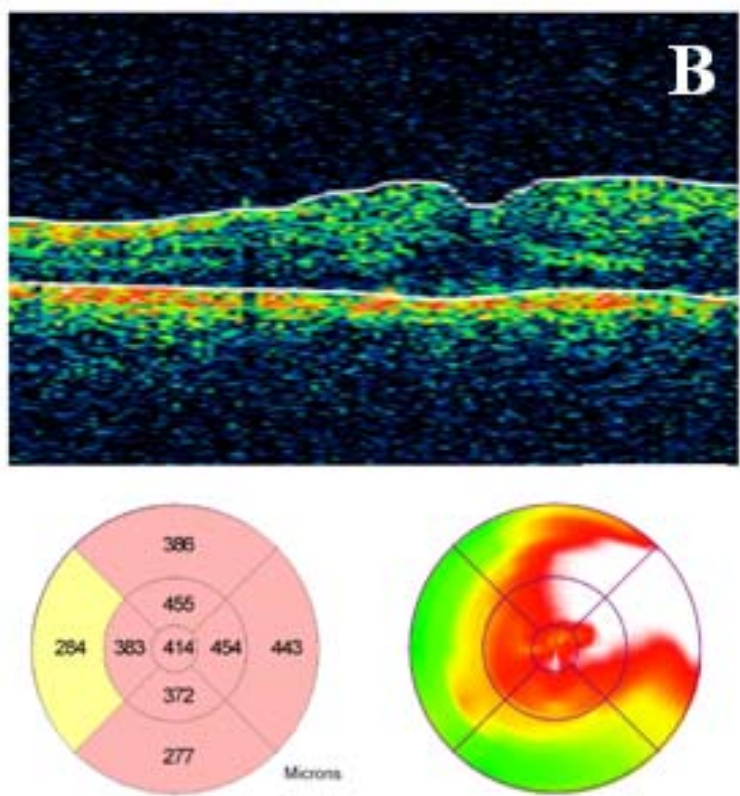

$\begin{array}{lllllllll}0 & 100 \quad 200 \quad 300 \quad 400 \quad 500 / m\end{array}$

\section{Figure 3. OCT readings at 0,24 , and 60 months.}

Fig. (3). (A) Optical coherence tomography of the right eye before intravitreal bevacizumab (Avastin) treatment showed significant cystoid macular edema. Central subfield thickness was $646 \mu \mathrm{m}$. (B) After treatment with Avastin (x4), the edema was substantially improved and retinal thickness was reduced to $414 \mu \mathrm{m}$. (C) At 60 months, edema remained largely resolved with retinal thickness at $424 \mu \mathrm{m}$.

term case series with follow-up periods up to 61 weeks [8]. They concluded that ranibizumab had minimal effects on most lesions but noted that efficacy was seen in their patient with the smallest lesion and the least exudation. Patients in their data set showed an average decrease of $9+/-20$ letters. Conversely, our patient experienced an improvement in VA of 25 letters while our patient's lesions initially improved then remained stable. Major differences between the two studies were the number of anti-VEGF injections administered and agent used; their patients had an average of $10.0+/-3.1$ injections of ranibizumab while our patient had 15 injections of bevacizumab. A single case study by Michels et al. also demonstrated slow regression of activity in hemangioblastoma lesions following ranibizumab treatment however follow-up was relatively short at 4 months and only involved 3 intravitreal injections. 
Another probable benefit of intermittent bevacizumab treatment appears to be the suppression of new hemangioblastomas. This subject was carefully assessed for such tumors throughout his follow up, and did not develop any additional hemangioblastomas in the peripheral retina, on fundus contact lens examination as well as with angiography of the periphery. Wong et al. observed an additional lesion arising de novo in only one out of five patients [8]. Documentation in the literature suggesting the untreated rate of new lesions arising does not explicitly exist. Chew described the course of several patients with ocular VHL disease who were not treated with anti-VEGF agents that developed additional peripheral lesions throughout their follow-up periods ${ }^{1}$. However, two patients that were treated with pegaptanib did not develop any new lesions during treatment or follow-up periods [1].

Based on subjects with age-related macular degeneration, where treatment with bevacizumab often shows excellent results, it appears that a similar benefit may apply to treatment with bevacizumab in cases of VHL associated hemangioblastomas. The structural changes due to the development of these retinal hemangioblastomas include large cells containing intractoplasmic lipid inclusions, reactive glial cells, as well as tumorlet cells [10]. Based on OCT findings, as well as clinical observation, it appears that VEGF suppression with bevacizumab does not appreciably affect these structural changes. Until more elegant solutions to VHL hemagioblastomas are developed, localized VEGF blocking therapy with bevacizumab (or ranibizumab) may be an interim solution for macular and optic nerve lesions.

\section{REFERENCES}

[1] Chew EY. Ocular manifestations of von Hippel-Lindau disease: clinical and genetic investigations. Trans Am Ophthalmol Soc 2005; 103: 495-511.

[2] Latif F, Tory K, Gnarra J, et al. Identification of the von HippelLindau disease tumor suppressor gene. Science 1993; 260: 131720.

[3] Seizinger BR, Rouleau GA, Ozelius LJ, et al. Von Hippel-Lindau disease maps to the region of chromosome 3 associated with renal cell carcinoma. Nature 1988; 332: 268-69.

[4] Singh AD, Shields CL, Shields JA. von Hippel-Lindau disease. Surv Ophthalmol 2001; 46: 117-42.

[5] De Klerk TA, Steel DH. Use of intravitreal bevacizumab in a patient with a Von Hippel-Lindau-associated retinal hemangioblastoma of the optic nerve head: a case report. K Med Case Rep 2008; 29(2): 182.

[6] Aiello LP, George DJ, Cahill MT, et al. Rapid and durable recovery of visual function in a patient with von Hippel-Lindau syndrome after systemic therapy with vascular endothelial growth factor receptor inhibitor SU5416. Ophthalmology 2002; 109: 174551.

[7] Dahr SS, Cusick M, Rodriguez-Coleman H, et al. Intravitreal antivascular endothelial growth factor therapy with pegaptanib for advanced von Hippel-Lindau disease of the retina. Retina 2007; 27: $150-8$.

[8] Wong WT, Liang KJ, Hammel K, et al. Intravitreal ranibizumab therapy for retinal capillary hemangioblastoma related to von Hippel-Landau disease. Ophthalmology 2008; 115(11): 1957-64.

[9] Michels, Messmer E, Sutter F, et al. Intravitreal anti-VEGF therapy for capillary hemangioblastomas in von Hippel-Landau disease. Klin Monatsbl Augenheilkd 2008; 225(4): 292-4.

[10] Chan CC, Daniel Collins AB and Chew EY. Molecular pathology of eyes with von Hippel-Lindau (VHL) disease. Retina 2007; 27: 17. 